\title{
Role of fine-needle aspiration cytology in evaluating thyroid nodules
}

\section{A retrospective study from a tertiary care center of Western region, Saudi Arabia}

Ahmed S. Bahaj, MD, JBORL, Haddad H. Alkaff, MD, ABORL, Bashair N. Melebari, MD, Anwar N. Melebari, MD, Suhail I. Sayed, MS, FACS, Syeddah S. Mujtaba, FCPS, Sherif K. Abdelmonim, MS, PhD, Saeed A. Alghamdi, MD, SBORL, Fares E. Alghamdi, MD, SBORL, OmarA.AbuSuliman, MD, SBORL, MohammadA. Alessa, MD, SBORL, FirasR. AbiSheffah, MD, ABORL, Anas H. Al-Tammas, MD, ABORL, Rajab A. Al-Zahrani, MD, SBORL, Osama A. Marglani, MD, FRCSC, John C. Heaphy, MD, ABOto, Osama A. Bawazir, MBBS, FRCSC, Ameen Z. Alherabi, FACS, FRCSC.

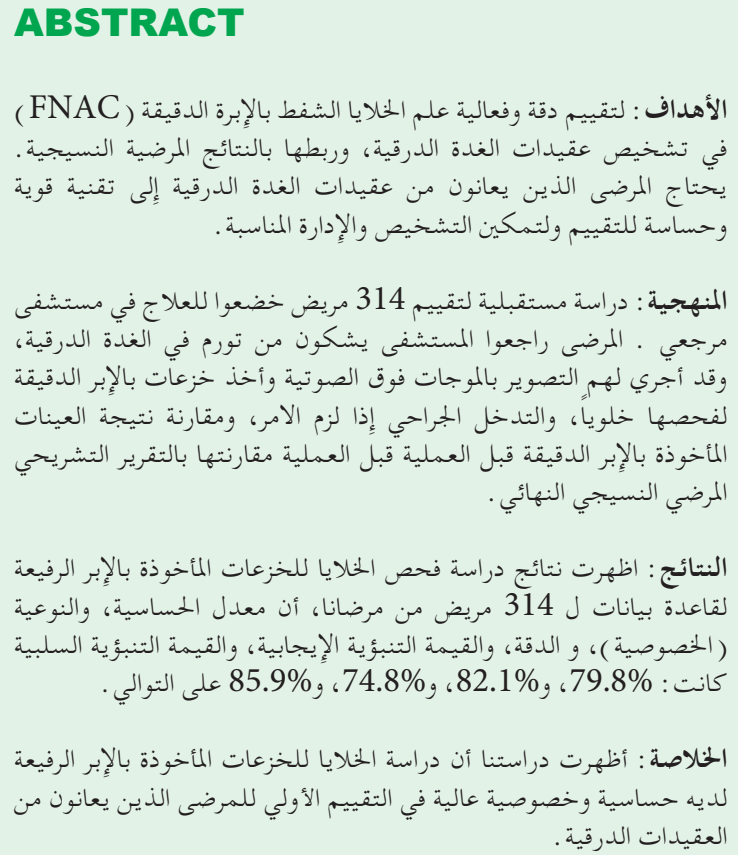

Objectives: To evaluate the accuracy and efficacy of fine-needle aspiration cytology (FNAC) in diagnosing thyroid nodules, correlating it with the histopathological findings.

Methods: A retrospective evaluation of 314 patients was undertaken at a tertiary referral center of King Abdullah Medical City (KAMC), Makkah, Kingdom of Saudi Arabia, between 2010-2019. Patients who presented with thyroid swellings underwent ultrasonography and FNAC. If indicated, surgery was performed. The FNAC findings were compared to the final histopathological reports.

Results: The findings for FNAC from our data set of 314 patients showed a sensitivity value of $79.8 \%$, specificity of $82.1 \%$, accuracy of $74.8 \%$, positive predictive value of $74.8 \%$, and negative predictive value of $85.9 \%$.

Conclusion: Our study showed that FNAC has high sensitivity and specificity in the initial evaluation of patients with thyroid nodules. When guided by ultrasonography, the accuracy can be markedly improved. Molecular markers once widely available can improve the diagnostic power of FNAC to be no less than the histopathologic evaluation of thyroid tissue.

Keywords: FNA, thyroid, carcinoma, cytology

Saudi Med J 2020; Vol. 41 (10): 1098-1103 doi: 10.15537/smj.2020.10.25417

From the Head and Neck \& Skull Base Surgery Center (Bahaj, Alkaff, Sayed, Abdelmonim, Alghamdi A, Alghamdi FE, Abu Suliman, Alessa, Al-Zahrani, Marglani, Alherabi); from the Department of Pathology (Mujtaba), King Abdullah Medical City, from the Department of Otolaryngology (BN. Melebari, AN. Melebari, Marglani, Alherabi), Head \& Neck Surgery; from the Department of Surgery (Bawazir), Pediatric Surgery Section, College of Medicine, Umm Al-Qura University, Makkah, from the Department of Otolaryngology-Head \& Neck Surgery (Abi Sheffah, Al-Tammas, Marglani, Heaphy, Alherabi); from the Department of Surgery (Bawazir), King Faisal Specialist Hospital \& Research Center, from the Department of OtolaryngologyHead \& Neck Surgery (Marglani, Alherabi), International Medical Center, Jeddah, from the Department of Otolaryngology-Head \& Neck Surgery (Al-Zahrani), Faculty of Medicine, Al-Baha University, Al-Baha, Kingdom of Saudi Arabia; and from the Department of Otolaryngology-Head \& Neck Surgery (Abdelmonim), Faculty of Medicine, Ain Shams University, Cairo, Egypt.

Received 16th April 2020. Accepted 13th September 2020.

Address correspondence and reprint request to: Dr. Ameen Z. Alherabi, Department of Otolaryngology-Head \& Neck Surgery, Umm Al-Qura University, Makkah, Kingdom of Saudi Arabia.E-mail: azherabi@uqu.edu.sa

ORCID ID: https://orcid.org/0000-0002-1001-3492 
T $\mathrm{n}$ recent times, the incidence of thyroid cancer has risen, with $10-15 \%$ of thyroid nodules being diagnosed with this pathology. ${ }^{1,2}$ Fine-needle aspiration cytology (FNAC) is currently employed in evaluating thyroid nodules which harbor malignancy. Fine-needle aspiration cytology is of pivotal diagnostic importance for clinicians in evaluating thyroid nodules and aids in preventing unindicated thyroid surgeries. ${ }^{3,4}$ Also, when combined with the Bethesda system for reporting thyroid cytopathology (TBSRTC), FNAC provides a more robust, reliable, and cost-effective diagnostic outcome. . $^{5-7}$

Therefore, it is the gold standard for diagnosing thyroid nodules. The efficacy of FNAC is debated due to its false-negative rates, which ranges from $1-15 \% .{ }^{8,9}$ Similarly, the role of FNAC in evaluating indeterminate cytology thyroid nodules is still being evaluated. ${ }^{10}$ Therefore, we conducted a retrospective cohort study to evaluate the accuracy and efficacy of FNAC in diagnosing thyroid nodules, correlating it with the histopathological findings.

Methods. This study is a retrospective evaluation of a prospectively maintained data set of outdoor patients who was presented with thyroid-related diseases at a tertiary care center of King Abdullah Medical City (KAMC), Makkah, Kingdom of Saudi Arabia (KSA) in the 10 years period of 2010-2019. The study was approved by the Institutional Review Board of KAMC, Makkah, KSA, and the National BioMedical Ethics Committee, King Abdulaziz City for Science and Technology (Protocol number 14-07-1433, registration number H-02-K-001). For patients who underwent FNAC in our institution, written informed consent was obtained from each patient prior to the FNAC procedure. A data collection form was used to collect the following information: age, gender, FNAC results, and histopathology results. The histopathology diagnosis of each patient was also obtained. Patients with no available records were excluded.

Ultrasonography (USG). Thyroid USG was performed by experienced board-certified radiologists with more than 10 years of experience in reporting thyroid ultrasounds. The USG was performed using a Philips L12-3 Broadband Linear array transducer with a

Disclosure. This study was funded by the Institute of Consulting Research \& Studies, Umm Al-Qura University, Makkah, Kingdom of Saudi Arabia. frequency of 3-14Mhz (ACUSON Sequoia Ultrasound Systems, Germany). Ultrasound-guided FNAC was performed according to the American thyroid association (ATA) guidelines 2009 \& 2015 guidelines, for the patients having the following features on USG: i) nodules with $>1$ suspicious feature; microcalcifications (stippled rim calcifications), hypoechogenicity, distorted nodular architecture, irregular margins and extrathyroidal extension (ETE). ii) suspicious cervical lymph nodes, iii) family history of thyroid malignancy, iv) change in voice/dysphagia/compressive symptoms, and v) past history of neck irradiation. For patients with multinodular goiter or the presence of multiple nodules, USG was used to detect the most concerning nodule and FNAC was performed under USG guidance. ${ }^{11}$

Fine-needle aspiration cytology. Under ultrasound guidance; a 23-gauge needle was used with a $20 \mathrm{ml}$ disposable syringe, and a minimum of 3 tissue passes was carried out to obtain satisfactory samples (each sample had 6 groups of benign follicular cells with 10 cells per group). All thyroid FNA samples were prepared by conventional methods using air-dried smears stained by a Romanowsky-type stain/ diff-quick stain or $95 \%$ ethanol-fixed smears stained by Papanicolaou stain. Thyroid cytology was reported using the 6-tier diagnostic categories introduced by the Bethesda system, which includes: non-diagnostic or unsatisfactory (Bethesda I), benign (Bethesda II), atypia of undetermined significance or follicular lesion of undetermined significance (Bethesda III), follicular neoplasm or suspicious for a follicular neoplasm (Bethesda IV), suspicious for malignancy (Bethesda V), and malignant (Bethesda VI) (Figure 1). ${ }^{9}$

Treatment. Patients were treated according to the American thyroid association (ATA) 2009 guidelines and surgery was considered for patients with i) Bethesda II-III with suspicious USG features of malignancy, ii) Bethesda IV-VI, iii) presence of stridor/dysphagia/ compressive symptoms, iv) presence of metastatic neck nodes, and v) patient preference. During the histopathological evaluation, multiple sections of the specimen were stained with hematoxylin and eosin (H\&E).

Statistical analysis. The Statistical Package for Social Sciences, version 22 (IBM Corp, Armonk, NY, USA) was used to analyze the data. The variables of the participants were estimated using descriptive statistics, including frequency counts and percentages for categorical variables. The Chi-square test was used to assess the significance of the relationship between categorical variables. A $p$-value of $<0.05$ with a $95 \%$ confidence interval was considered significant. 

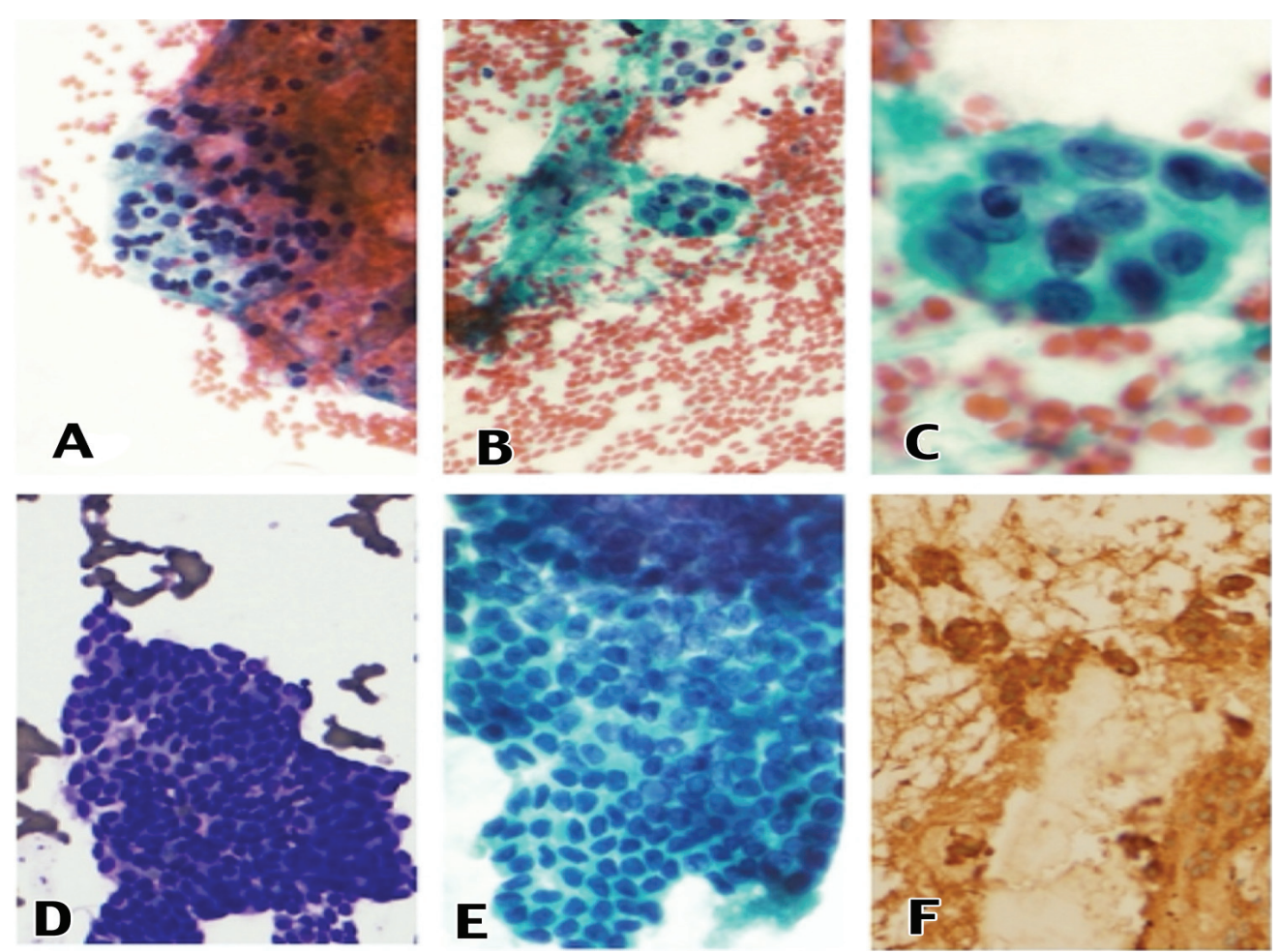

Figure 1 - Papanicolaou stain of direct smear showing follicular cells in macrofollicular arrangement, A) 20X (Bethesda II). Papanicolaou stain showing follicular cells with mild overlapping and nuclear enlargement, B) 20X (Bethesda III). Nuclear enlargement and occasional nuclear grooving, C) 40X (Bethesda III). Diff-quick staining (DQ) showing follicular cells in microfollicular pattern, follicular neoplasm, D) 20X (Bethesda IV). Follicular cells showing nuclear grooving, psammoma bodies and overlapping, E) 40X (Bethesda VI). Tumor cells with positive staining for calcitonin, F) 20X (Medullary thyroid carcinoma).

Sensitivity, true positive rate (TPR), specificity, true negative rate (TNR), and accuracy were calculated after analyzing the final histopathology report.

Results. Three hundred fourteen thyroid patients with thyroid nodules were initially evaluated by FNAC. Subsequently, these underwent a post-surgical evaluation using histopathological methods. Amongst our patient, there were 258 (81\%) females and 56 (19\%) males, with a male-to-female ratio of 1:4.6. The mean age was $42.3 \pm 7.3$ years. On the final histopathology, 150 nodules were found to be malignant. These included papillary carcinoma, medullary carcinoma, poorly differentiated carcinoma, Hurthle cell carcinoma, and anaplastic carcinoma. We found 164 nodules to be of benign pathology. These included colloid nodular goiter, follicular lesion, Hurthle cell lesion, and Hashimoto's thyroiditis. (Figure 2).

The TBSRTC reporting of the thyroid nodules was performed and is depicted in Figure 3. Fine-needle aspiration cytology was repeated in 100 nodules for the following reasons: non-diagnostic/unsatisfactory cytological findings ( $8 / 8$ nodules), benign cytology (28/152 nodules), atypia of undetermined significance (51/51 nodules), and follicular neoplasm (13/13 nodules). The histopathological findings of the thyroid nodules are shown in Table 1.

The sensitivity, specificity, positive likelihood ratio, negative likelihood ratio, positive predictive value, negative predictive value, and accuracy of FNAC are shown in Table 2. There were no major or minor complications such as hematoma, tumor seeding along the needle track, or infection reported in our patient data set during the FNAC procedure.

Discussion. Thyroid cancer incidence has been increasing, mainly due to its rising awareness among the population as well as improvements in the diagnostic modalities. ${ }^{11}$ Cancers of the thyroid have the best prognosis as compared to cancers of the other sites of the head and neck region. ${ }^{11}$ Additionally, with improved overall survival, the need to provide the best outcomes 


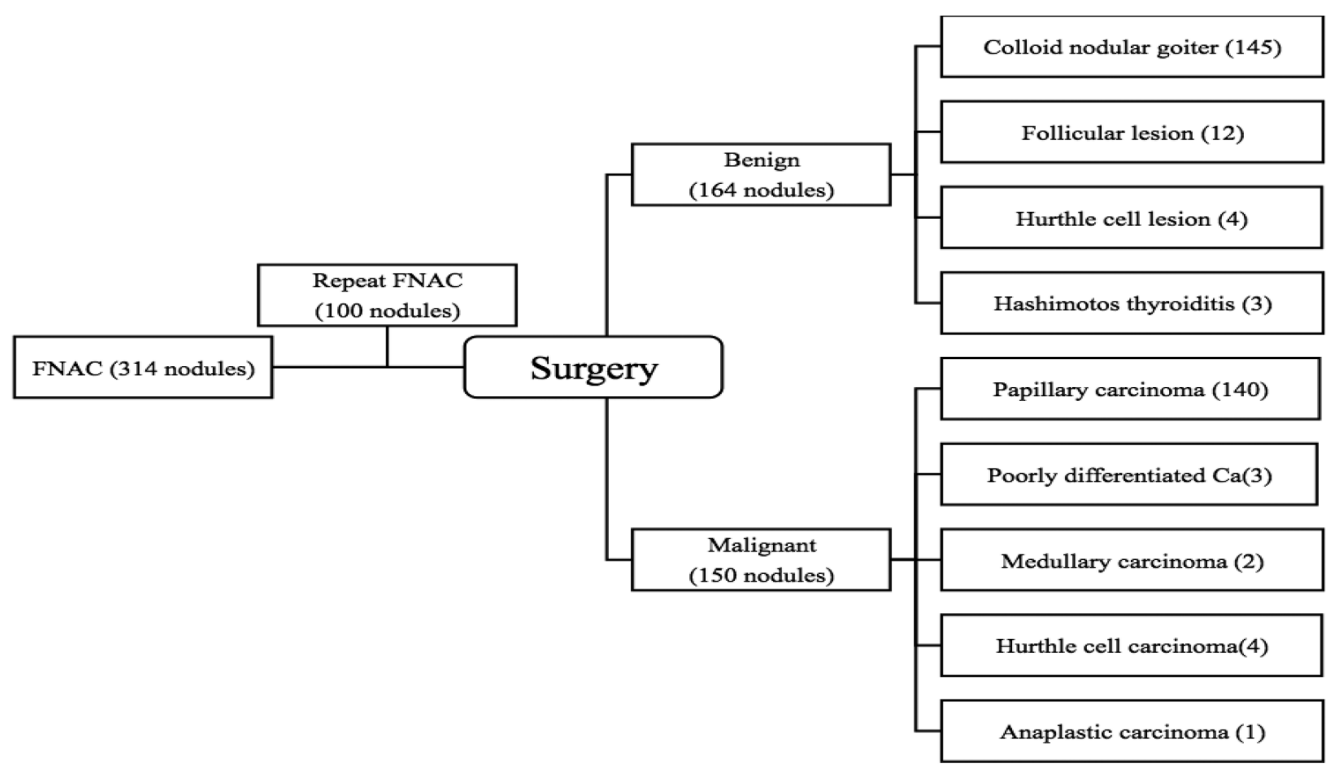

Figure 2 - Schematic representation of the histopathology reporting of thyroid nodules in the patient population.

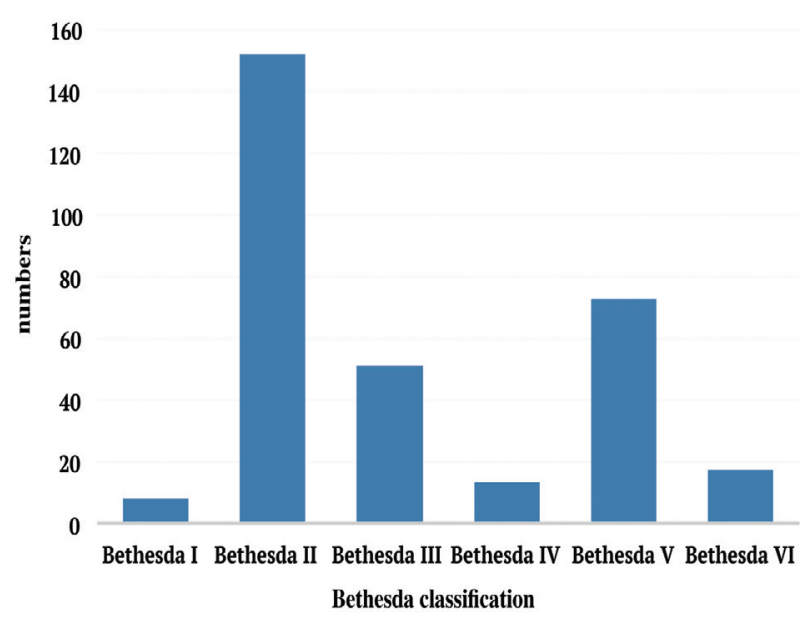

Figure 3 - Fine-needle aspiration cytology reporting of the 314 thyroid nodules according to the Bethesda System for reporting thyroid cytopathology.

for these patients is of utmost importance. Hence, there is a need for proper diagnostic workup of these tumors to individualize the therapeutic modalities.

Currently, the majority of patients presenting with thyroid nodules undergo USG examination. Based on the USG findings, patients are subsequently subjected to FNAC. ${ }^{12}$ Ultrasonography guided FNAC is currently the method of choice for evaluating thyroid nodules because of its high sensitivity. Additionally, the TBSRTC has greatly improved the uniformity in reporting thyroid cytology. ${ }^{12}$ However, FNAC has several short
Table 1 - Diagnostic outcome of the thyroid nodule evaluation using fineneedle aspiration cytology as compared to the histopathology results $(p<0.001)$.

\begin{tabular}{lrrrrr}
\hline FNAC (Bethesda classification) & \multicolumn{4}{c}{$\begin{array}{c}\text { Histopathological examination } \\
\text { Benign }\end{array}$} \\
& N & & \multicolumn{2}{c}{ Malignant } \\
& 8 & 6 & $(75.0)$ & $2(25.0)$ \\
\hline Bethesda I & 152 & 131 & $(86.2)$ & $21(13.8)$ \\
Bethesda II & 51 & 16 & $(31.4)$ & $35(68.6)$ \\
Bethesda III & 13 & 7 & $(53.9)$ & $6(46.2)$ \\
Bethesda IV & 73 & 4 & $(5.5)$ & $69(94.5)$ \\
Bethesda V & 17 & 0 & $(0)$ & $17(100)$ \\
Bethesda VI & 314 & 164 & $(52.0)$ & $150(48.0)$ \\
Total & & &
\end{tabular}

FNAC: fine-needle aspiration cytology

comings; it can present false-negative cytology and is operator dependent. ${ }^{13}$ Therefore, we planned this study to understand whether FNAC results guide us in managing patients with thyroid nodules.

In our study, the mean population age was 42.3 years, with a range from $23-78$ years, which was in accordance with previous studies reported in the literature. ${ }^{14,15}$ Female patients predominated our data set as compared to males ( $81 \%$ vs. $19 \%$ ), whereas in the literature, the prevalence in females is reported in the range of $71-86 \% .^{16,17}$ 
Table 2 - Diagnostic accuracy of FNAC in evaluating the thyroid nodules.

\begin{tabular}{lcc}
\hline FNAC & Value & $95 \%$ CI \\
\hline Sensitivity & $79.8 \%$ & $70.8-87$ \\
Specificity & $82.1 \%$ & $75-87.7$ \\
Positive likelihood ratio & 4.45 & $3.14-6.30$ \\
Negative likelihood ratio & 0.25 & $0.17-0.36$ \\
Positive predictive value & $74.8 \%$ & $67.6-80.8$ \\
Negative predictive value & $85.9 \%$ & $80.5-90$ \\
Accuracy & $81.2 \%$ & $75.9-85.7$ \\
\hline
\end{tabular}

FNAC: fine-needle aspiration cytology, CI: confidence interval

The rate of non-diagnostic test namely, Bethesda I in our series was $2.5 \%$ which is in concordance with the reported literature (1.6-20\%). ${ }^{18,19}$ Although, it has been recommended to keep the non-diagnostic rate to be less than $10 \% .{ }^{19}$ We had to repeat FNAC in 100/314 nodules to decrease the chances of false-negative findings. It has been shown in the published literature that factors such as small nodule size, mixed solid cystic nodules, deep-seated nodules, and inexperienced technicians lead to poor sampling of the FNAC. ${ }^{20}$ Similarly, thyroid nodules which are sclerotic and show extensive calcification along with cystic changes, it is better to repeat the FNAC to avoid false-negative findings. ${ }^{20,21}$ The practice of repeated FNAC can sometimes also lead to undue stress to patients and may not be cost-effective. ${ }^{21}$ In our data set, repeated FNAC led to improved diagnosis and better decision making in the Bethesda III category, wherein we were able to upstage 10/51 nodules into the Bethesda V category. Ultrasonography guided FNAC was used in all patients as it led to better diagnostic yield. ${ }^{12}$ Although we did not find any change after repeating FNAC for Bethesda I and II, this is in accordance with the published literature. ${ }^{20}$

Our FNAC data set comprised $48.4 \%$ benign cytology and $5.4 \%$ malignant cytology. We did not include AUS/FLUS category (16.2\%) as well as suspicious for malignancy (23\%) into the malignant category. The literature had reported these categories into intermediate category but we have considered them as separate categories for better understanding of the results. ${ }^{19}$ Furthermore, histopathological evaluation revealed a slight increase in the diagnosis of benign pathology $(52.2 \%)$, whereas there was a significant increase in malignant pathology (47.7\%). This significant increase is mostly due to the upstaging seen in Bethesda IV and V to malignant pathology on the final histopathological diagnosis. Papillary carcinoma (93.3\%) was the most common tumor, followed by Hurthle cell carcinoma (2.6\%), poorly differentiated carcinoma (2\%), medullary carcinoma (1.3\%), and anaplastic carcinoma (0.6\%). This is in accordance with the study by Afroze et al, ${ }^{17}$ wherein papillary carcinoma was the most common thyroid tumor reported on final histopathology. ${ }^{21,22}$

A study by Roy et $\mathrm{al}^{23}$ showed that the sensitivity of FNAC was $81.5 \%$ and specificity of FNAC was $95.3 \%$ in their evaluation of thyroid nodules. ${ }^{23}$ Sengupta et $\mathrm{al},{ }^{24}$ had a sensitivity of $90 \%$ and specificity of $100 \%$. Gupta et $\mathrm{al}^{25}$ in their evaluation of thyroid nodules by FNAC, had a sensitivity of $80 \%$ and specificity of $86.6 \%$. In our data set, we had a sensitivity of $79.8 \%$ and specificity of $82.5 \%$. Furthermore, correlating the FNAC and histopathological findings in our data set, the diagnostic accuracy was $81.15 \%$. Roy et $\mathrm{al}^{23}$ reported $91.96 \%$ accuracy, Sengupta et $\mathrm{al}^{24}$ was $98.3 \%$, and Gupta et $\mathrm{a}^{25}$ was $83 \%$ accuracy in their respective studies (Table 3). ${ }^{26}$

Study limitations. Our study has several limitations such as, the retrospective setting in a single-center can lead to selection bias. Some patients did not opt for surgery. Hence, although FNAC was performed for those patients, we did not include them in our study. To counter these

Our study has a large sample size and is a singlecenter study. Uniform standard protocols were carried out in evaluating the thyroid nodules.

Table 3 - Comparison between the present study and the reported literature.

\begin{tabular}{lcccccc}
\hline $\begin{array}{l}\text { Study } \\
\text { (year) }\end{array}$ & $\begin{array}{c}\text { Number } \\
\text { of } \\
\text { patients }\end{array}$ & $\begin{array}{c}\text { Sensitivity } \\
(\%)\end{array}$ & $\begin{array}{c}\text { Specificity } \\
(\%)\end{array}$ & $\begin{array}{c}\text { PPV } \\
(\%)\end{array}$ & $\begin{array}{c}\text { NPV } \\
(\%)\end{array}$ & $\begin{array}{c}\text { Accuracy } \\
(\%)\end{array}$ \\
\hline $\begin{array}{l}\text { Roy et al } \\
(2019)^{23}\end{array}$ & 112 & 81.5 & 95.3 & 84.6 & 94.2 & 91.96 \\
$\begin{array}{l}\text { Sengupta et } \\
\text { al (2011) }\end{array}$ & 178 & 90.0 & 100 & 100 & 98.8 & 98.3 \\
$\begin{array}{l}\text { Gupta et al } \\
(2010)^{25}\end{array}$ & 75 & 80.0 & 86.6 & 80 & 86.6 & 84.0 \\
$\begin{array}{l}\text { Kessler et } \\
\text { al (2005) }\end{array}$ & 170 & 79.0 & 98.5 & 76.6 & 98.7 & 92.0 \\
$\begin{array}{l}\text { Present } \\
\text { study }\end{array}$ & 314 & 79.8 & 82.05 & 74.77 & 84.91 & 81.2 \\
\hline
\end{tabular}

PPV: positive predictive values, NPV: negative predictive values 
In conclusion, FNAC is considered the gold standard with high yield and cost-effective tool in the preoperative evaluation of thyroid nodules and, when combined with USG, has high sensitivity and specificity in providing useful information in guiding the management of thyroid nodules. Although it is a safe, reproducible, and cost-effective method, it can result in false-negative reports. In the future, FNAC will be further improved through the use of molecular tests. This should enhance its diagnostic yield and guide us further in the management of thyroid lesions.

Acknowledgment. The authors gratefully acknowledge Editage, (www.editage.com) for English language editing.

\section{References}

1. Cooper DS, Doherty GM, Haugen BR, Kloos RT, Lee SL, Mandel SJ, et al. Revised American Thyroid Association Management Guidelines for patients with thyroid nodules and differentiated thyroid cancer. Thyroid 2009; 19: 1167-1214.

2. Yeung MJ, Serpell JW. Management of the solitary thyroid nodule. Oncologist 2008; 13: 105-112.

3. Hagag P, Strauss S, Weiss M. Role of ultrasound-guided fine-needle aspiration biopsy in evaluation of nonpalpable thyroid nodules. Thyroid 1998; 8: 989-995.

4. Cibas ES, Baloch ZW, Fellegara G, LiVolsi VA, Raab SS, Rosai $\mathrm{J}$, et al. A prospective assessment defining the limitations of thyroid nodule pathologic evaluation. Ann Intern Med 2013; 159: 325-332.

5. Hegedüs L. Clinical practice. The thyroid nodule. N EnglJ Med 2004; 351: 1764-1771.

6. Amrikachi M, Ramzy I, Rubenfeld S, Wheeler TM. Accuracy of fine-needle aspiration of thyroid. Arch Pathol Lab Med 2001; 125: 484-488.

7. Tysome JR, Chandra A, Chang F, Puwanarajah P, Elliott $\mathrm{M}$, Caroll $\mathrm{P}$, et al. Improving prediction of malignancy of cytologically indeterminate thyroid nodules. Br J Surg 2009; 96: 1400-1405.

8. Sinna EA, Ezzat N. Diagnostic accuracy of fine needle aspiration cytology in thyroid lesions. J Egypt Natl Canc Inst 2012; 24: 63-70.

9. Liu X, Medici M, Kwong N, Angell TE, Marqusee E, Kim MI, et al. Bethesda categorization of thyroid nodule cytology and prediction of thyroid cancer type and prognosis. Thyroid 2016; 26: 256-261.

10. Sclabas GM, Staerkel GA, Shapiro SE, Fornage BD, Sherman SI, Vassillopoulou-Sellin R, et al. Fine-needle aspiration of the thyroid and correlation with histopathology in a contemporary series of 240 patients. Am J Surg 2003; 186: 702-709.

11. Shin HY, Jee YH, Cho ER. Body mass index and incidence of thyroid cancer in Korea: the Korean cancer prevention study-II. J Cancer Res Clin Oncol 2017; 143: 143-149.
12. Haugen BR, Alexander EK, Bible KC, Doherty GM, Mandel SJ, Nikiforov YE, et al. 2015 American thyroid association management guidelines for adult patients with thyroid nodules and differentiated thyroid cancer: The American thyroid association guidelines task force on thyroid nodules and differentiated thyroid cancer. Thyroid 2016; 26: 1-133.

13. Lee SH, Kim MH, Bae JS, Lim DJ, Jung SL, Jung CK. Clinical outcomes in patients with non-diagnostic thyroid fine needle aspiration cytology: usefulness of the thyroid core needle biopsy. Ann Surg Oncol 2014; 21: 1870-1877.

14. Bakhos R, Selvaggi SM, DeJong S, Gordon DL, Pitale SU, Herrmann M, et al. Fine-needle aspiration of the thyroid: rate and causes of cytohistopathologic discordance. Diagn Cytopathol 2000; 23: 233-237.

15. Morgan JL, Serpell JW, Cheng MS. Fine-needle aspiration cytology of thyroid nodules: how useful is it? ANZ J Surg 2003; 73: 480-483.

16. Kamal MM, Arjune DG, Kulkarni HR. Comparative study of fine needle aspiration and fine needle capillary sampling of thyroid lesions. Acta Cytol 2002; 46: 30-34.

17. Afroze N, Kayani N, Hasan SH. Role of fine needle aspiration cytology in the diagnosis of palpable thyroid lesions. Indian J Pathol Microbiol 2002; 45: 241-246.

18. Wang CC, Friedman L, Kennedy GC, Wang H, Kebebew E, Steward DL, et al. A large multicenter correlation study of thyroid nodule cytopathology and histopathology. Thyroid 2011; 21: 243-251.

19. Ali SZ. Thyroid cytopathology: Bethesda and beyond. Acta Cytol 2011; 55: 4-12.

20. Menéndez Torre E, Pineda Arribas J, Martínez de Esteban JP, López Carballo MT, de Miguel C, Salvador P. Value of repeated fine needle aspiration cytology in patients with nodular goiter. Acta Cytol 2007; 51: 850-852.

21. Merchant FH, Hirschowitz SL, Cohan P, Van Herle AJ, Natarajan S. Simultaneous occurrence of medullary and papillary carcinoma of the thyroid gland identified by fine needle aspiration. A case report. Acta Cytol 2002; 46: 762-766.

22. Graciano AJ, Chone CT, Fischer CA, Bublitz GS, Peixoto AJ. [Repeated fine-needle aspiration cytology for the diagnosis and follow-up of thyroid nodules]. Braz J Otorbinolaryngol 2014; 80: 422-427. [Portuguese].

23. Roy PK, Bandyopadhyay S, Dubey AB, Sengupta A. A comparative study on aspiration cytology and histopathology in diagnosis of thyroid nodule and its correlation. Indian J Otolaryngol Head Neck Surg 2019; 71: 997-1001.

24. Sengupta A, Pal R, Kar S, Zaman FA, Sengupta S, Pal S. Fine needle aspiration cytology as the primary diagnostic tool in thyroid enlargement. J Nat Sci Biol Med 2011; 2: 113-118.

25. Gupta M, Gupta S, Gupta VB. Correlation of fine needle aspiration cytology with histopathology in the diagnosis of solitary thyroid nodule. J Thyroid Res 2010; 2010: 379051.

26. Kessler A, Gavriel H, Zahav S, Vaiman M, Shlamkovitch N, Segal S, et al. Accuracy and consistency of fine-needle aspiration biopsy in the diagnosis and management of solitary thyroid nodules. Isr Med Assoc J 2005; 7: 371-373. 